\title{
Evaluation of Release Kinetics and Mechanisms of Curcumin and Curcumin- $\beta$-Cyclodextrin Inclusion Complex Incorporated in Electrospun Almond Gum/PVA Nanofibers in Simulated Saliva and Simulated Gastrointestinal Conditions
}

\author{
Atefe Rezaei $^{1,2}$ (D) Ali Nasirpour ${ }^{3}$
}

Published online: 27 March 2019

(C) The Author(s) 2019

\begin{abstract}
Curcumin and curcumin- $\beta$-cyclodextrin (CD) inclusion complex were successfully encapsulated in almond gum/polyvinyl alcohol (PVA) nanofibers using electrospinning procedure. The release mechanisms and kinetics of encapsulated curcumin and curcumin- $\beta C D$ inclusion complex were evaluated in simulated gastrointestinal and simulated saliva conditions. The release data were fitted to zero order, Kopcha, Korsmeyer-Peppas, and Higuchi models to evaluate release mechanisms and kinetics. The controlled release of curcumin in simulated gastrointestinal and simulated saliva conditions showed that the nanofibers containing complex had the higher release in comparison of only curcumin at all time points. This can be related to the higher solubility of curcumin incorporated into the $\beta \mathrm{CD}$. The release profile of curcumin and its complex were similar. Release kinetics of curcumin and its complex in both simulated gastrointestinal and simulated saliva conditions followed a Fickian diffusion mechanism. The release data were well fitted with Kopcha, Korsmeyer-Peppas, and Higuchi models with $R^{2}$ values higher than 0.9176 .
\end{abstract}

Keywords Curcumin $\cdot$ Almond gum nanofiber $\cdot \beta$-cyclodextrin $\cdot$ Inclusion complex $\cdot$ Controlled release

\section{Introduction}

Curcumin is a natural colorant which is obtained from the rhizome of the herb Curcuma longa. Curcumin is a hydrophobic polyphenol and has many food and pharmaceutical applications. In the food industry, curcumin can be used as a natural colorant and also as a stabilizer in jellies. Moreover, it can be used as an artificial colorant replacer in cheeses, yogurts,

Atefe Rezaei

atefe.rezaei@nutr.mui.ac.ir

Ali Nasirpour

ali.nasirpour@cc.iut.ac.ir

1 Department of Food Science and Technology, School of Nutrition and Food Science, Isfahan University of Medical Sciences, Isfahan, Iran

2 Food Security Research Center, Isfahan University of Medical Sciences, Isfahan, Iran

3 Department of Food Science and Technology, College of Agriculture, Isfahan University of Technology, Isfahan 84156-83111, Iran cereals, ice creams, mustards, pickles, and soups [1]. In the pharmaceutical industry, curcumin has many properties including anti-inflammatory, antibacterial, antioxidant, anticancer, anti-Alzheimer, anticoagulant, antiparasitic, and antimutagenic [2-5].

The application of curcumin is restricted in the food industry and pharmaceutical formulation due to its poor solubility and low stability in different conditions. Curcumin has high sensitivity to light, thermal treatment, alkaline conditions, oxygen, enzyme, metallic ions and ascorbic acid [6]. To overcome these limitations, encapsulation is a suitable technique. Several delivery systems have been used for encapsulation of curcumin such as nanoparticles [7, 8], nanofibers [9, 10], liposomes [11], and yeast cells [1]. Complexation with $\mathrm{CD}$ is another approach for increasing the solubility of curcumin $[5,12,13]$. CDs are cyclic oligosaccharides with six $(\alpha \mathrm{CD})$, seven $(\beta C D)$, or eight $(\gamma \mathrm{CD})$ glucose units and have a hydrophobic cavity and a hydrophilic shell. The hydrophobic cavity entraps a wide variety of lipophilic compounds without any covalent bonds. Among three CDs, $\beta C D$ can better fit with different molecules with molecular weights between 200 and $800 \mathrm{~g} / \mathrm{mol}$ 
[14]. Curcumin has two phenyl rings such that each of them can be incorporated into one $\mathrm{CD}$ and forms an inclusion complex. Therefore, the molar ratio of curcumin: CD is 1:2.

Nanofibers are the good delivery system for encapsulation. Nanofibers have high encapsulation efficiency and high surface area per unit volume that make them suitable for controlled release applications [15]. Electrospinning is a simple and versatile technique for producing nanofibers using high electric field. The encapsulation of curcumin in different polymer nanofibers has been studied by several researchers. Sampath et al (2014) encapsulated curcumin in poly (lactic-co-glycolic) acid nanofibers and used them for the treatment of carcinoma [16]. More recently, researchers encapsulated curcumin in amaranthpullulan electrospun nanofibers and observed a controlled and sustained release of curcumin in buffer solution $(\mathrm{pH}=7.4)$ during an in vitro digestion process [9].

Almond gum is a highly soluble polysaccharide that has high molecular weight and is suitable for electrospinning. Electrospun almond gum nanofibers are a good choice for preserving and increasing the stability of sensitive compounds $[17,18]$.

Incorporation of curcumin into a hydrophilic polymer will increase its solubility, stability, and finally lead to the enhanced bioavailability. Other researchers also confirmed the efficiency of polysaccharides such as Arabic gum [19], hyaluronic acid (HA) [20], and alginate [21] for conjugation with curcumin and indicated that the solubility of curcumin has been improved.

The purpose of this study is investigating the influence of almond gum nanofibers and inclusion complex of $\beta C D$ on curcumin release under simulated gastrointestinal and simulated saliva conditions. Also, the mechanisms and kinetics of curcumin release will be studied in simulated gastrointestinal and simulated saliva media.

\section{Materials and Methods}

\subsection{Materials}

Almond gum exudates were gathered from the trunk and branches of almond trees (Amygdalus communis L.) in Saman, Chaharmahal, and Bakhtiari Province, Iran. Almond gum has two fractions: water soluble fraction and water insoluble fraction. The water soluble fraction of almond gum with $M_{\mathrm{w}}=15.9 \times 10^{3} \mathrm{KDa}, M_{\mathrm{n}}=5.02 \times 10^{3} \mathrm{KDa}$ and $\mathrm{PDI}=3.16$ was used for electrospinning [18]. $\beta C D$ was purchased from SD-fine corporation, India. Polyvinyl alcohol (PVA) $\left(M_{\mathrm{w}}\right.$ : 145000 , fully hydrolyzed) and curcumin were purchased from Merck (Merck, Germany). Moreover, sodium hydroxide, potassium dihydrogen phosphate, sodium chloride, sodium bicarbonate, potassium chloride, and hydrochloric acid were purchased from Merck (Darmstadt, Germany). $\alpha$-Amylase (from fungal, $120000 \mathrm{SKB}$ ) was purchased from ORBA (Istanbul, Turkey).

\subsection{Preparation of Electrospinning Solution}

At first, the inclusion complex of curcumin and $\beta C D$ was prepared by freeze drying method described by Mohan et al (2012) with a few modifications [2]. Briefly, curcumin $(0.25 \mathrm{~g})$ and $\beta C D(1.54 \mathrm{~g})$ were dissolved in ethanol $(10 \mathrm{ml})$ and water $(30 \mathrm{ml})$, respectively and then the solution of curcumin added dropwise into the $\beta C D$ solution. The mixture solution was stirred for 7 days at $37{ }^{\circ} \mathrm{C}$ and then filtered through $0.45 \mu \mathrm{m}$ filter. The clear solution was freeze dried at $-40{ }^{\circ} \mathrm{C}$ for $24 \mathrm{~h}$ (Dena Vacuum, Iran).

For the fabrication of electrospinning solution, the solutions of almond gum/PVA ( 7 and $8 \% w / w)$ in the proportion of 80:20 $(w / w)$ were prepared. At first, the determined amount of PVA was added to deionized water and stirred at $80{ }^{\circ} \mathrm{C}$ for $2 \mathrm{~h}$. Then, almond gum was added to the PVA solution and stirred for $1 \mathrm{~h}$ at $45^{\circ} \mathrm{C}$. The obtained solution was hydrated overnight in a refrigerator. The specified amounts of curcumin and curcumin- $\beta C D$ inclusion complex were added to this solution and stirred for $8 \mathrm{~h}$ at room temperature (Table 1). Finally, the solution was left to stabilize for $1 \mathrm{~h}$ before electrospinning.

\subsection{Electrospinning Process and Characterization}

The electrospinning solutions were loaded into a 1-ml syringe (needle inner diameter $=0.8 \mathrm{~mm}$ ) and fixed in a horizontal position onto a syringe pump. Electrospinning was performed at ambient conditions $\left(25^{\circ} \mathrm{C}\right.$ and $30 \%$ relative humidity) using the following parameters: the applied voltage was $18 \mathrm{kV}$, the flow rate was $0.125 \mathrm{ml} / \mathrm{h}$ and nozzle-collector distance was $15 \mathrm{~cm}$.

The morphology and diameter of obtained nanofibers were analyzed by a field emission scanning electron microscope (FESEM). The samples were gold-sputtered prior to imaging under argon (HITACHI S-4160, Korea). The average diameter of nanofibers was calculated by analyzing of around 60 fibers in the FESEM images using Digimizer software.

\subsection{Loading Efficiency of Curcumin Incorporated in Almond Gum/PVA Nanofibers}

To determine the loading efficiency of curcumin in the almond gum/PVA nanofibers, approximately $10 \mathrm{mg}$ of the nanofibers were added to $5 \mathrm{ml}$ of $50 \%$ ethanol aqueous solution and agitated for $24 \mathrm{~h}$ at room temperature to completely dissolve [22]. The absorbance of the samples was read at $425 \mathrm{~nm}$ after filtration through a $0.45 \mu \mathrm{m}$ filter [23, 24]. The amount of curcumin presented in the samples was calculated using calibration curves. The 
Table 1 The composition of electrospinning solutions, average fiber diameter and loading efficiency of obtained nanofibers

\begin{tabular}{llllll}
\hline $\begin{array}{l}\text { Concentration } \\
\text { of almond gum/ } \\
\text { PVA }(\% w / w)\end{array}$ & $\begin{array}{l}\text { Concentration } \\
\text { of curcumin } \\
(\% w / w)\end{array}$ & $\begin{array}{l}\text { Concentration } \\
\text { of complex } \\
(\% w / w)\end{array}$ & $\begin{array}{l}\text { Average } \\
\text { fiber } \\
\text { diameter } \\
(\mathrm{nm})\end{array}$ & Fiber morphology & $\begin{array}{l}\text { Loading } \\
\text { efficiency } \\
(\%)\end{array}$ \\
\hline 8 & 1 & - & $169 \pm 35$ & Bead-free nanofibers & $75 \pm 0.20$ \\
8 & 2 & - & $151 \pm 28$ & Bead-free nanofibers & $65 \pm 0.75$ \\
8 & 3 & - & $121 \pm 31$ & Nanofibers with beads & - \\
7 & 4 & - & $127 \pm 29$ & Nanofibers with beads & - \\
8 & - & 2 & $134 \pm 30$ & Bead-free nanofibers & $92 \pm 0.50$ \\
8 & - & 3 & $125 \pm 24$ & Bead-free nanofibers & $94 \pm 0.85$ \\
7 & - & 4 & $98 \pm 18$ & Bead-free nanofibers & $95 \pm 0.25$ \\
7 & - & 5 & $118 \pm 23$ & Nanofibers with beads & - \\
\hline
\end{tabular}

nanofibers without curcumin were used as blank. The loading efficiency was calculated according to Eq. 1:

Loading efficiency (\%)

$=\frac{\text { Calculated curcumin concentration from the standard curve }}{\text { Theoretical curcumin concentration }} \times 100$

\subsection{In Vitro Curcumin Release}

Curcumin release was studied in simulated gastrointestinal and simulated saliva conditions at $37^{\circ} \mathrm{C}$ and $100 \mathrm{rpm}$ in a shaking thermostatic incubator. The gastrointestinal release mediums were the gastric solution $(\mathrm{pH}=1.2)$ for stomach and phosphate buffer $(\mathrm{pH}=6.8)$ for intestine.

The gastric solution was prepared by dissolving $2.0 \mathrm{~g}$ of sodium chloride in $80 \mathrm{ml}$ of $1 \mathrm{M}$ hydrochloric acid and then water was added up to $1000 \mathrm{ml}$. The $\mathrm{pH}$ of this solution was 1.2. For the preparation of gastric solution, purified pepsin also is needed but if hard gelatin capsules are not being tested, pepsin can be often deleted for simplifying its preparation. The phosphate buffer was prepared by dissolving $6.8 \mathrm{~g}$ of monobasic potassium phosphate in $250 \mathrm{ml}$ of water and then added $77 \mathrm{ml}$ of $0.2 \mathrm{M}$ sodium hydroxide and water up to $1000 \mathrm{ml}$. The final $\mathrm{pH}$ was adjusted to 6.8 using $0.2 \mathrm{~N}$ sodium hydroxide or $0.2 \mathrm{~N}$ hydrochloric acid. Similar to gastric solution for preparation of phosphate solution, pancreatin also is needed but if hard gelatin capsules are not being tested, pancreatin can be often deleted for simplifying its preparation [25, 26]. The simulated saliva was prepared using Davis et al. method [27]. Simulated saliva consisted of $25.0 \mathrm{meq} / 1$ sodium bicarbonate, $20 \mathrm{meq} / 1$ potassium chloride, $2.0 \mathrm{meq} / 1$ sodium chloride and $2 \mathrm{~g} / \mathrm{l} \alpha$-amylase. The final $\mathrm{pH}$ was adjusted to 7.0.

To study the curcumin release in simulated gastrointestinal medium, around of $15 \mathrm{mg}$ of the nanofibers containing curcumin and $35 \mathrm{mg}$ of the nanofibers containing complex were added separately into the $40 \mathrm{ml}$ of gastric solution and agitated continuously for $2 \mathrm{~h}$ and subsequently the nanofibers were subjected to the $50 \mathrm{ml}$ of intestinal buffer for $6 \mathrm{~h}$. Also, $15 \mathrm{mg}$ of the nanofibers containing curcumin and $35 \mathrm{mg}$ of the nanofibers containing complex were added into the $200 \mathrm{ml}$ of simulated saliva separately. At predetermined time intervals, $5 \mathrm{ml}$ of the solution was removed for sampling and replaced with equal volume of the release medium to maintain a constant volume. After filtration through a $0.45-\mu \mathrm{m}$ filter, the absorbance of the samples was recorded at a wavelength of $425 \mathrm{~nm}$ by a UV spectrophotometer. The amount of released curcumin was plotted as the percentage release versus time. All measurements were conducted in triplicate, and results are reported as average values \pm S.D.

The cumulative amount $(\%)$ of released curcumin from nanofibers was calculated using the following Eq. (2):

Curcumin release $(\%)$

$$
=\frac{\text { The amount of released curcumin }}{\text { Total amount of curcumin in the nanofibers }} \times 100(2)
$$

\subsection{Release Kinetics}

The curcumin release data were kinetically evaluated by zeroorder (Eq. (3)), Kopcha (Eq. (4)), Korsmeyer-Peppas (Eq. (5)) and Higuchi (Eq. (6)) models. The release data fitting was done using Matlab software (R2007) (data fitting toolbax) and after evaluating the regression coefficient, the best kinetic model was obtained. $M_{\mathrm{t}}$ in all equations is the amount of curcumin released at time $t$.

Zero-order model : $M_{\mathrm{t}}=k \mathrm{t}$,

where $k$ is the zero order release constant [28].

Kopcha model : $\mathrm{M}_{\mathrm{t}}=\mathrm{A} \times \mathrm{t}^{0.5}+\mathrm{B} \times \mathrm{t}$,

where $\mathrm{A}$ and $\mathrm{B}$ are the diffusion rate and the erosion rate constants, respectively. The main release mechanism is related to the ratio of $\mathrm{A} / \mathrm{B}$. If $\mathrm{A} / \mathrm{B}$ is higher than 1 , the main release 
mechanism is controlled by diffusion and if it is lower than 1 , it is controlled by erosion. In the case of being equal to 1 , both diffusion and erosion are important for release mechanism [29].

Korsmeyer-Peppas model : $\mathrm{M}_{\mathrm{t}}=\mathrm{kt}^{\mathrm{n}}$,

where $k$ is Korsmeyer-Peppas constant which is related to the properties of the delivery system and the encapsulated substance. The $n$ exponent is the release exponent that shows the curcumin release mechanism: for $n<0.5$, a pseudo-Fickian diffusion mechanism; $n=0.5$ a Fickian mechanism; $0.5<n<1$, an anomalous diffusion mechanism; and for $n=1$, a nonFickian diffusion mechanism controls the mechanism of release $[30,31]$.

Higuchi model : $M_{\mathrm{t}}=\mathrm{k} \times \mathrm{t}^{0.5}$,

where $k$ is Higuchi constant [32].

\section{Results and Discussion}

\subsection{FESEM Analysis}

Electrospinning of different solutions of almond gum/PVA/ curcumin and almond gum/PVA/complex solutions were done according to Table 1 . The solutions containing 1 or $2 \%(w / w)$ of curcumin and also 2, 3, or $4 \%(w / w)$ of complex, produced proper and bead-free nanofibers whereas the solutions containing 3 or $4 \%(w / w)$ curcumin and $5 \%(w / w)$ complex did not produce uniform nanofibers. The production of nanofibers with beads from almond gum/PVA/curcumin solution is due to the insolubility of curcumin in almond gum/PVA/curcumin solution which leads to the aggregation of spinning solution. Also, for nanofibers containing complex, the high viscosity of the almond gum/PVA/complex solutions leads to the production of nanofibers with beads. The FESEM images of almond gum/PVA/curcumin nanofibers and almond gum/PVA/complex nanofibers which have uniform nanofibers are presented in Fig. 1. Electrospinning of almond gum/PVA $8 \%(w / w)$ with $1 \%(w / w)$ of curcumin produced smooth and bead-free nanofibers with $169 \pm 35 \mathrm{~nm}$ diameter. Furthermore, almond gum/ PVA 7\% $(w / w)$ with $4 \%(w / w)$ complex produced bead-free nanofibers with $98 \pm 18 \mathrm{~nm}$ diameter. These two nanofibers indicated higher loading efficiency compared to other producing nanofibers according to Table 1 . Therefore, these nanofibers were selected for studying the curcumin release in simulated gastrointestinal and simulated saliva media.

\subsection{Loading Efficiency}

The loading efficiency of curcumin in almond gum/PVA/ curcumin nanofibers and almond gum/PVA/complex nanofibers is presented in Table 1. As can be seen, the loading efficiency of curcumin in almond gum/PVA/curcumin nanofibers decreased from 75 to $65 \%$ by increasing the amount of curcumin from $1 \%(w / w)$ to $2 \%(w / w)$. This can be explained by the low solubility of curcumin. Indeed, the curcumin precipitation may occur during the electrospinning process by increasing the curcumin concentration. Other researchers also obtained similar results that, by increasing the amount of curcumin in zein nanofibers, the loading efficiency is decreased [33].

The polymer which used for encapsulation of curcumin has important effect on its loading efficiency. Using zein electrospun fibers as carrier, researchers obtained the loading efficiency of $77-83 \%$ [34] and $82.4-86.7 \%$ [35] for curcumin. Some researchers also reported high loading efficiency for curcumin encapsulated in polyvinylpyrrolidone nanoparticles $(99.3 \%)$ [36] and pure gelatin nanofibers (around of 95\%) [22].

For the nanofibers containing complex, the loading efficiency was around of $92-95 \%$ and was higher than the samples containing only curcumin. This is due to the higher solubility of curcumin in complex with $\mathrm{CD}$ that leads to higher stability of electrospinning solution. Moreover, it was observed that by increasing the amount of curcumin complex in nanofibers loading efficiency slightly increased. These results indicated that almost $100 \%$ of complexed curcumin was protected after being incorporated into almond gum/PVA nanofibers.

\subsection{In Vitro Release}

As can be seen in Fig. 2a, for curcumin release in simulated gastrointestinal media, both nanofibers containing curcumin and complex showed initial burst release in the first $120 \mathrm{~min}$ (gastric condition) around of 27 and 50\%, respectively. The burst release can be related to the surface-loaded curcumin molecules which have weak bound with fiber surface and also have high diffusion tendency. The interaction of bioactive compound with soultion and also the properties of solution have important effect on distribution of bioactive compound in the nanofibers. Most of the bioactive compounds may exposure to charge repulsion and dispense on the surface of nanofibers [37]. The release followed by sustained release over $480 \mathrm{~min}$. Nanofibers containing complex showed higher release (around of $85 \%$ ) compared with nanofibers containing curcumin (around of 70\%) after $480 \mathrm{~min}$. This can be related to the higher solubility of incorporated curcumin into the $\mathrm{CD}$. The release of curcumin from nanofibers is limited due to its low solubility. However, inclusion complex with CD increases its aqueous solubility. CD has a surfactant-like properties which induced the higher dissolution of the complex [38]. Also, CD can decrease the interfacial tension between the curcumin and almond gum/PVA solution which leads to higher dissolution and curcumin release from nanofibers [39]. 
Fig. 1 FESEM image of (a) almond gum/PVA (80:20) 8\% (w/ $w)+$ curcumin $1 \%(w / w)(\mathbf{b})$ almond gum/PVA $(80: 20) 7 \%(w /$ $w)+$ complex $4 \%(w / w)$, voltage $=18 \mathrm{kV}$, nozzle-collector distance $=15$,

flow rate $=0.15 \mathrm{ml} / \mathrm{h}$

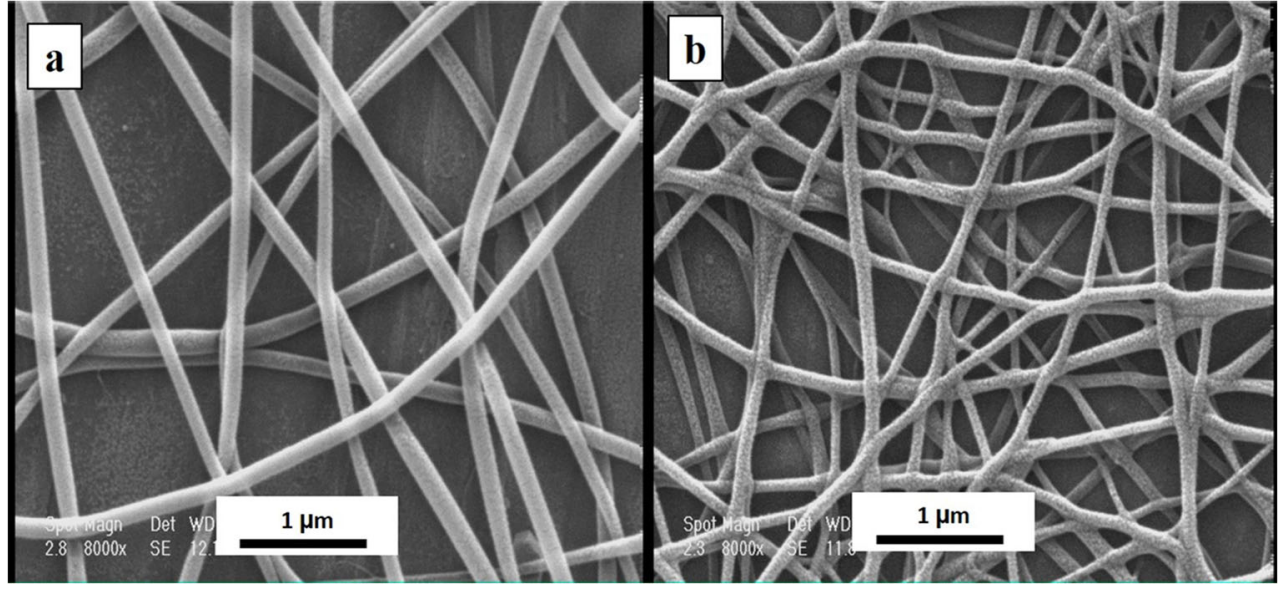

For simulated saliva conditions, nanofibers containing complex exhibited higher release in comparison of nanofibers containing only curcumin at all time points (Fig. 2b). The cumulative release of curcumin and complex were 57 and $75 \%$ after $180 \mathrm{~min}$, respectively. As previously mentioned, the higher release of curcumin in nanofibers containing complex is due to the higher solubility of curcumin.

Other researchers obtained the similar results that curcumin/cyclodextrin inclusion complex/polylactic acid nanofibers released curcumin more than curcumin/polylactic acid nanofibers at $\mathrm{pH} 1$ and $\mathrm{pH}$ 7.4. This is due to the solubility enhancement of curcumin after inclusion complexation with cyclodextrin [40]. Sedghi and Shaabani (2016) also encapsulated curcumin into the core-shell PVA/chitosan fibers in order to control the curcumin release. Curcumin indicated a burst release in phosphate buffered saline medium (around of $40 \%$ of the loaded curcumin) at the first hours due to the surface-loaded curcumin molecules following a constant slow release (up to $85 \%$ ) for 10 days [41].

Mutlu et al. (2018) studied the release of curcumin from the electrospun poly (3-hydroxy butyric acid-co-3-hydroxy valeric acid) (PHBV) nanofibers in phosphate-buffered saline (PBS) solution (at $\mathrm{pH} 7.4 \pm 0.2$ ). Their results showed that approximately $25 \%$ of the curcumin was released in first $30 \mathrm{~min}$; and after $200 \mathrm{~min}$, the curcumin released in nanofibers containing $0.1,0.3$, and $0.5 \%(w / v)$ of curcumin was $45 \%$, $63 \%$, and $78 \%$, respectively [42].

\subsection{Release Kinetics}

In order to study the mechanism of curcumin release from almond gum/PVA nanofibers, the release data were fitted to various kinetic models (zero order, Kopcha, KorsmeyerPeppas, and Higuchi). The modeling results are presented in Table 2 . The release data were fitted adequately with Kopcha, Korsmeyer-Peppas, and Higuchi models with $R^{2}$ values higher than 0.9176 .
To characterize the release mechanism, the value of $n$ as release exponent in Korsmeyer-Peppas model was calculated. The $n$ value was higher than 0.5 for nanofibers containing curcumin in gastrointestinal conditions and nanofibers containing curcumin and complex in simulated saliva conditions. This confirms that anomalous diffusion controls the curcumin release from almond gum/ PVA nanofibers. However, for nanofibers containing complex in gastrointestinal conditions, the $n$ value was 0.47 which shows that pseudo-Fickian diffusion controls the curcumin release.

However, the release exponent in Korsmeyer-Peppas gives a little information about the release mechanism and therefore the ratio of $\mathrm{A} / \mathrm{B}$ in Kopcha model was determined. As can be seen in Table 2, this ratio, in all media, is higher than 1 which indicated Fickian diffusion is the main release mechanism.

Sampath and coworkers (2014) also studied the in vitro release kinetic and mechanism of curcumin loaded in poly (lactic-co-glycolic) acid nanofibers in phosphate buffer at $\mathrm{pH}$ 7.4. They indicated that the release profile was fitted to Korsmeyer-Peppas model and the release mechanism was a combination of surface drug dissolution with nonFickian-diffusion [16]. Wang et al (2017) studied the release of curcumin from zein nanofibers in Milli-Q water medium and showed that the predominant release of curcumin from nanofibers was Fickian diffusion and diffusion behavior well described with first-order model and Hixson-Crowell model diffusion behavior [33]. Sun et al (2013) showed the release of curcumin and curcumin-CD complex from PVA nanofibers mainly governed by diffusion mechanism and were well fitted to Higuchi model [43]. It is important to mention that almond gum is a water soluble polymer and can absorb water at high moisture condition and swell. The swelling of nanofibers leads to cleavage of interaction between nanofibers and curcumin that helps to release and diffuse curcumin from nanofibers. 
Fig. 2 The release of curcumin and complex in (a) simulated gastrointestinal and (b) simulated saliva conditions
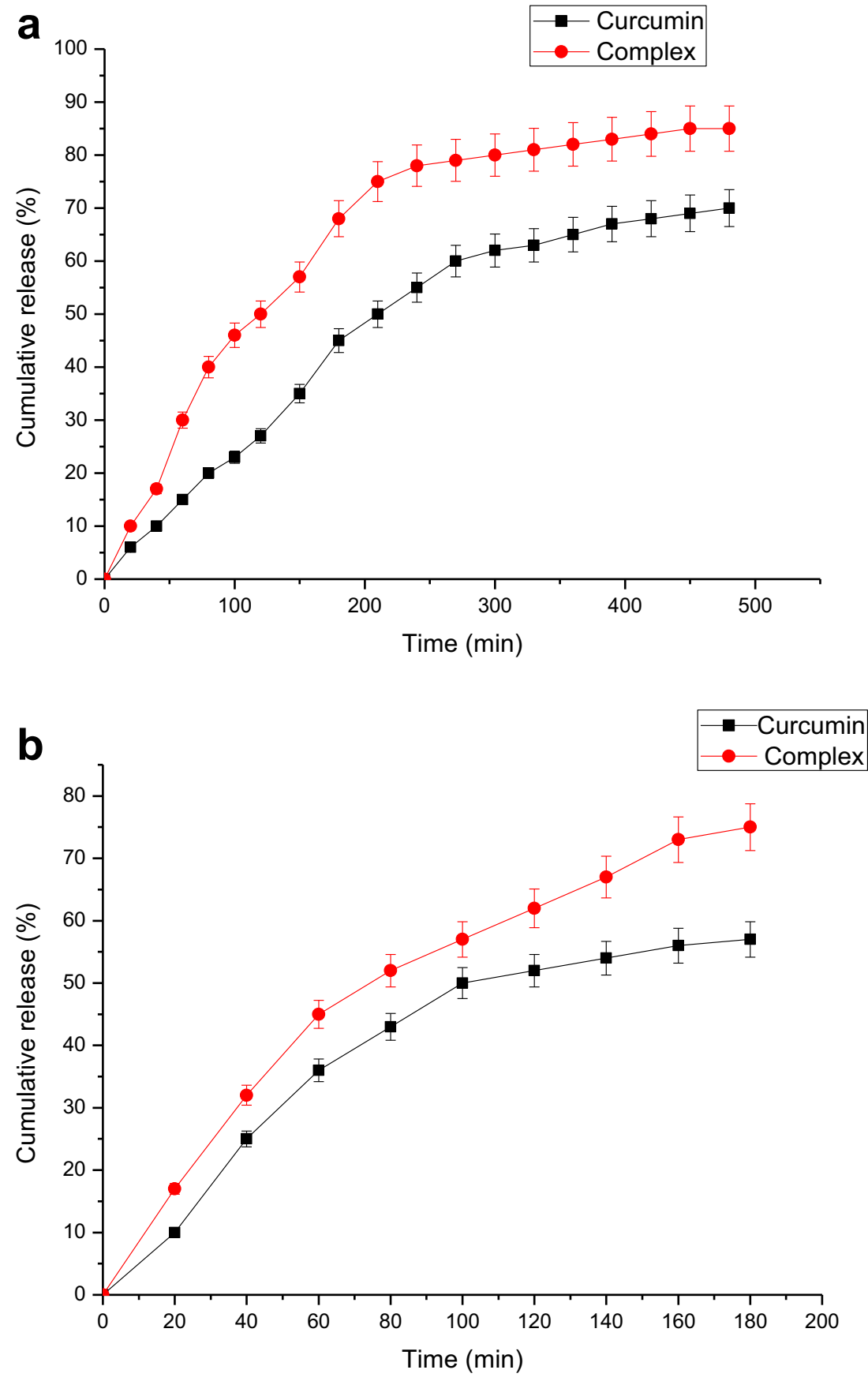

Table 2 Model parameters of curcumin release in different release conditions

\begin{tabular}{|c|c|c|c|c|c|c|c|c|c|c|c|}
\hline \multirow[t]{2}{*}{ Release condition } & \multirow[t]{2}{*}{ Formulation } & \multicolumn{2}{|c|}{ Zero order } & \multicolumn{3}{|c|}{ Kopcha model } & \multicolumn{3}{|c|}{ Korsmeyer-Peppas } & \multicolumn{2}{|c|}{ Higuchi } \\
\hline & & K & $R^{2}$ & A & $\mathrm{B}$ & $R^{2}$ & K & $N$ & $R^{2}$ & K & $R^{2}$ \\
\hline Simulated gastrointestinal & Pure curcumin & 0.1799 & 0.8768 & 2.117 & 0.0648 & 0.959 & 1.292 & 0.6625 & 0.9657 & 3.255 & 0.9341 \\
\hline Simulated gastrointestinal & Complex & 0.236 & 0.5866 & 5.112 & -0.0417 & 0.9471 & 5.19 & 0.47 & 0.9411 & 4.379 & 0.9393 \\
\hline Simulated saliva & Pure curcumin & 0.3932 & 0.7966 & 25.72 & -24.85 & 0.9176 & 3.4 & 0.5588 & 0.9587 & 4.498 & 0.9545 \\
\hline Simulated saliva & Complex & 0.4928 & 0.8389 & 4.879 & 0.0677 & 0.9876 & 4.075 & 0.5678 & 0.9894 & 5.626 & 0.984 \\
\hline
\end{tabular}




\section{Conclusion}

In this work, almond gum/PVA nanofibers containing curcumin and curcumin- $\beta$-cyclodextrin inclusion complex were successfully prepared by electrospinning process. The release study of curcumin incorporated in almond gum/PVA nanofibers were accomplished in simulated gastrointestinal and simulated saliva conditions. Curcumin showed a rapid release in simulated gastric conditions due to the surface loading and then followed a sustained release. In simulated saliva conditions, nanofibers containing complex exhibited higher release in comparison of nanofibers containing only curcumin at all time points. This is related to the higher solubility of curcumin after complexation with $\mathrm{CD}$. In order to investigate the kinetics of curcumin release from nanofibers, the release data were fitted by zero-order, Kopcha, Korsmeyer-Peppas, and Peppas-Sahlin models. The results indicated that the release data were fitted well to the Kopcha, Korsmeyer-Peppas, and Higuchi models with $R^{2}$ values higher than 0.9176. The curcumin release was mainly controlled by diffusion mechanism in all medium.

Open Access This article is distributed under the terms of the Creative Commons Attribution 4.0 International License (http:// creativecommons.org/licenses/by/4.0/), which permits unrestricted use, distribution, and reproduction in any medium, provided you give appropriate credit to the original author(s) and the source, provide a link to the Creative Commons license, and indicate if changes were made.

\section{References}

1. Paramera, E., Konteles, S., \& Karathanos, V. (2011). Microencapsulation of curcumin in cells of Saccharomyces cerevisiae. Food Chemistry, 125, 892-902.

2. Mohan, P., Sreelakshmi, G., Muraleedharan, C., \& Joseph, R. (2012). Water soluble complexes of curcumin with cyclodextrins: characterization by FT-Raman spectroscopy. Vibrational Spectroscopy, 62, 77-84.

3. Singh, R., Tonnesen, H. H., Vogensen, S. B., Loftsson, T., \& Másson, M. (2010). Studies of curcumin and curcuminoids. XXXVI. The stoichiometry and complexation constants of cyclodextrin complexes as determined by the phase-solubility method and UV-vis titration. Journal of Inclusion Phenomena and Macrocyclic Chemistry, 66, 335-348.

4. Yallapu, M., Jaggi, M., \& Chauhan, S. (2010). b-Cyclodextrincurcumin self-assembly enhances curcumin delivery in prostate cancer cells. Colloids and Surfaces B: Biointerfaces, 79, 113-125.

5. Ntoutoume, G. M. A. N., Granet, R., Mbakidi, J. P., Brégier, F., Léger, D. Y., Fidanzi-Dugas, C., Lequart, V., Joly, N., Liagre, B., Chaleix, V., \& Sol, V. (2015). Development of curcumin-cyclodextrin/cellulose nanocrystals complexes: new anticancer drug delivery systems. Bioorganic \& Medicinal Chemistry Letters, 26, 941-945.

6. Mangolim, C. S., Moriwaki, C., Nogueira, A. C., Sato, F., Baesso, M. L., Neto, A. M., \& Matioli, G. (2014). Curcumin-b- cyclodextrin inclusion complex: stability, solubility, characterisation by FT-IR, FT-Raman, X-ray diffraction and photoacoustic spectroscopy, and food application. Food Chemistry, 153, 361-370.

7. Dadhaniya, P., Patel, C., Muchhara, J., Bhadja, N., Mathuria, N., \& Vachhani, K. (2011). Safety assessment of a solid lipid curcumin particle preparation: acute and subchronic toxicity studies. Food and Chemical Toxicology, 49, 1834-1842.

8. Das, R. K., Kasoju, N., \& Bora, U. (2010). Encapsulation of curcumin in alginatechitosan-pluronic composite nanoparticles for delivery to cancer cells. Nanomedicine: Nanotechnology, Biology and Medicine, 6, 153-160.

9. Blanco-Padilla, A., Lopez-Rubio, A., Loarca-Pina, G., GomezMascaraque, L. G., \& Mendoza, S. (2015). Characterization, release and antioxidant activity of curcumin-loaded amaranthpullulan electrospun fibers. LWT - Food Science and Technology, 63, 1137-1144.

10. Hu, J., Zeng, F., Wei, J., Chen, Y., \& Chen, Y. (2014). Novel controlled drug delivery system for multiple drugs based on electrospun nanofibers containing nanomicelles. Journal of Biomaterials Science, Polymer, (3), 257-268.

11. Li, L., Braiteh, F., \& Kurzrock, R. (2005). Liposome-encapsulated curcumin. Cancer, 104, 1322-1331.

12. Dhule, S. S., Penfornis, P., Frazier, T., Walker, R., Feldman, J., Tan, G., He, J., Alb, A., John, V., \& Pochampally, R. (2012). Curcuminloaded $\gamma$-cyclodextrin liposomal nanoparticles as delivery vehicles for osteosarcoma. Nanomedicine: Nanotechnology, Biology, and Medicine, 8, 440-451.

13. Popat, A., Karmakar, S., Jambhrunkar, S., Xu, C., \& Yu, C. (2014). Curcumin-cyclodextrin encapsulated chitosan nanoconjugates with enhanced solubility and cell cytotoxicity. Colloids and Surfaces B: Biointerfaces, 117, 520-527.

14. Szente, L., \& Szejtli, J. (2004). Cyclodextrins as food ingredients. Trends in Food Science \& Technology, 15, 137-142.

15. Rezaei, A., Nasirpour, A., \& Fathi, M. (2015). Application of cellulosic nanofibers in food science using electrospinning and its potential risk. Comprehensive Reviews in Food Science and Food Safety, 14, 269-284.

16. Sampath, M., Lakra, R., Korrapati, P., \& Sengottuvelan, B. (2014). Curcumin loaded poly (lactic-co-glycolic) acid nanofiber for the treatment of carcinoma. Colloids and Surfaces B: Biointerfaces, $117,128-134$.

17. Rezaei, A., Tavanai, H., \& Nasirpour, A. (2016). Fabrication of electrospun almond gum/PVA nanofibers as a thermostable delivery system for vanillin. International Journal of Biological Macromolecules, 91, 536-543.

18. Rezaei, A., Nasirpour, A., \& Tavanai, H. (2016). Fractionation and some physicochemical properties of almond gum (Amygdalus communis L.) exudates. Food Hydrocolloids, 60, 461-469.

19. Sarika, P. R., James, N. R., Kumar, P. R. A., Raj, D. K., \& Kumary, T. V. (2015). Gum arabic-curcumin conjugate micelles with enhanced loading for curcumin delivery to hepatocarcinoma cells. Carbohydrate Polymers, 134, 167-174.

20. Manju, S., \& Sreenivasan, K. (2011). Conjugation of curcumin onto hyaluronic acid enhances its aqueous solubility and stability. Journal of Colloid and Interface Science, 359(1), 318-325.

21. Dey, S., \& Sreenivasan, K. (2014). Conjugation of curcumin onto alginate enhances aqueous solubility and stability of curcumin. Carbohydrate Polymers, 99, 499-507.

22. Deng, L., Kang, X., Liu, Y., Feng, F., \& Zhang, H. (2017). Effects of surfactants on the formation of gelatin nanofibres for controlled release of curcumin. Food Chemistry, 231, 70-77.

23. Taylor, S., \& McDowell, I. (1992). Determination of the curcuminoid pigments in turmeric (Curcuma domestica Val) by reversed-phase high-performance liquid chromatography. Chromatographia, 34, 74-77. 
24. Carvalhd, D. M., Takeuchi, K. P., Geraldine, R. M., Moura, C. J., \& Torres, M. C. L. (2015). Production, solubility and antioxidant activity of curcumin nanosuspension. Food Science and Technology, 35(1), 115-119.

25. Galia, E., Nicolaides, E., Horter, D., Lobenberg, R., Reppas, C., \& Dressman, J. B. (1998). Evaluation of various dissolution media for predicting in vivo performance of class I and II drugs. Pharmaceutical Research, 15(5), 698-705.

26. Stippler, E., Kopp, S., \& Dressman, J. B. (2004). Comparison of US pharmacopeia simulated intestinal fluid TS (without pancreatin) and phosphate standard buffer $\mathrm{pH} 6.8$, TS of the international pharmacopoeia with respect to their use in in vitro dissolution testing. Dissolution Technologies. https://doi.org/10.14227/DT110204P6.

27. Davis, R., Hartman, C., \& Fincher, J. (1971). Dialysis of ephedrine and pentobarbital from whole human saliva and simulated saliva. Journal of Pharmaceutical Sciences, 60(3), 429-432.

28. Donbrow, M., \& Samuelov, Y. (1980). Zero order drug delivery from double-layered porous films: release rate profiles from ethyl cellulose, hydroxypropyl cellulose and polyethylene glycol mixtures. Journal of Pharmacy and Pharmacology, 32(7), 463-470.

29. Kopcha, M., Lordi, N., \& Tojo, K. (1991). Evaluation of release from selected thermosoftening vehicles. The Journal of Pharmacy and Pharmacology, 43(6), 382-387.

30. Korsmeyer, R. W., Gurny, R., Doelker, E., Buri, P., \& Peppas, N. A. (1983). Mechanism of solute release from porous hydrophilic polymers. International Journal of Pharmaceutical Sciences, 15, 2535.

31. Li, N., Fu, C., \& Zhang, L. (2014). Using casein and oxidized hyaluronic acid to form biocompatible composite hydrogels for controlled drug release. Material Science Engineering $C$, Materials for Biological Application, 36, 287-293.

32. Higuchi, T. (1961). Rate of release of medicaments from ointment bases containing drugs in suspension. Journal of Pharmaceutical Sciences, 50(10), 874-875.

33. Wang, H., Hao, L., Wang, P., Chen, M., Jiang, S., \& Jiang, S. (2017). Release kinetics and antibacterial activity of curcumin loaded zein fibers. Food Hydrocolloids, 63, 437-446.

34. Brahatheeswaran, D., Mathew, A., Aswathy, R. G., Nagaoka, Y., Venugopal, K., Yoshida, Y., Maekawa, T., \& Sakthikumar, D. (2012). Hybrid fluorescent curcumin loaded zein electrospun nanofibrous scaffold for biomedical applications. Biomedical Materials, 7, 1-16.
35. Yilmaz, A., Bozkurt, F., Cicek, P. K., Dertli, E., Durak, M. Z., \& Yilmaz, M. T. (2016). A novel antifungal surface-coating application to limit postharvest decay on coated apples: molecular, thermal and morphological properties of electrospun zein-nanofiber mats loaded with curcumin. Innovative Food Science and Emerging Technologies, 37, 74-83.

36. Yen, F. L., Wu, T. H., Tzeng, C. W., Lin, L. T., \& Lin, C. C. (2010). Curcumin nanoparticles improve the physicochemical properties of curcumin and effectively enhance its antioxidant and antihepatoma activities. Journal of Agricultural and Food Chemistry, 58, 73767382.

37. Rezaei, A., Fathi, M., \& Jafari, S. M. (2019). Nanoencapsulation of hydrophobic and low-soluble food bioactive compounds within different nanocarriers. Food Hydrocolloids, 88, 146-162.

38. Lin, S., \& Kao, Y. (1989). Solid particulates of drug $\beta$-cyclodextrin inclusion complexes directly prepared by spray drying technique. International Journal of Pharmaceutics, 56, 249-259.

39. Guyot, M., Fawaz, F., Bildet, J., Bonini, F., \& Lagueny, A. M. (1995). Physicochemical characterization and dissolution of norfloxacin/cyclodextrin inclusion compounds and PEG solid dispersions. International Journal of Pharmaceutics, 123, 53-63.

40. Aytac, Z., \& Uyar, T. (2017). Core-shell nanofibers of curcumin/ cyclodextrin inclusion complex and polylactic acid: enhanced water solubility and slow release of curcumin. International Journal of Pharmaceutics, 518, 177-184.

41. Sedghi, R., \& Shaabani, A. (2016). Electrospun biocompatible core/shell polymer-free core structure nanofibers with superior antimicrobial potency against multi drug resistance organisms. Polymer, 101, 151-157.

42. Mutlu, G., Calamak, S., Ulubayram, K., \& Guven, E. (2018). Curcumin-loaded electrospun PHBV nanofibers as potential wound dressing material. Journal of Drug Delivery Science and Technology, 43, 185-193.

43. Sun, X., Williams, G., Hou, X., \& Zhu, L. (2013). Electrospun curcumin-loaded fibers with potential biomedical applications. Carbohydrate polymer, 94, 147-153.

Publisher's Note Springer Nature remains neutral with regard to jurisdictional claims in published maps and institutional affiliations. 